ARTICLE

DOI: $10.1038 /$ ncomms7767 OPEN

\title{
Ultra-sensitive all-fibre photothermal spectroscopy with large dynamic range
}

Wei Jin ${ }^{1,2, \star}$, Yingchun Cao ${ }^{1,2, \star, \dagger}$, Fan Yang ${ }^{1,2, \star} \&$ Hoi Lut $\mathrm{Ho}^{1,2}$

Photothermal interferometry is an ultra-sensitive spectroscopic means for trace chemical detection in gas- and liquid-phase materials. Previous photothermal interferometry systems used free-space optics and have limitations in efficiency of light-matter interaction, size and optical alignment, and integration into photonic circuits. Here we exploit photothermalinduced phase change in a gas-filled hollow-core photonic bandgap fibre, and demonstrate an all-fibre acetylene gas sensor with a noise equivalent concentration of 2 p.p.b. $\left(2.3 \times 10^{-9}\right.$ $\mathrm{cm}^{-1}$ in absorption coefficient) and an unprecedented dynamic range of nearly six orders of magnitude. The realization of photothermal interferometry with low-cost near infrared semiconductor lasers and fibre-based technology allows a class of optical sensors with compact size, ultra sensitivity and selectivity, applicability to harsh environment, and capability for remote and multiplexed multi-point detection and distributed sensing.

\footnotetext{
${ }^{1}$ Department of Electrical Engineering and Photonics Research Center, The Hong Kong Polytechnic University, Hung Hom, Kowloon, Hong Kong, China. 2 Photonic Sensors Research Center, The Hong Kong Polytechnic University Shenzhen Research Institute, No. 18 Yuexing 1st Road, Nanshan District, Shenzhen 518057, China. * These authors contributed equally to this work. † Present address: Department of Electrical and Computer Engineering, Rice University, 6100 Main Street, Houston, Texas 77005, USA. Correspondence and requests for materials should be addressed to W.J. (email: eewjin@polyu.edu.hk).
} 
$\mathrm{D}$ etection of trace chemicals sensitively and selectively is important for environmental, safety and industrial process monitoring as well as national security applications. Laser absorption spectroscopy (LAS), which relies on the 'finger-print' absorption lines of molecules for identifying and detecting trace chemicals, is a powerful technique that offers high selectivity and sensitivity ${ }^{1-4}$. The employment of optical fibrebased technologies greatly expands the capability of LAS for remote interrogation, space limited and harsh environmental applications, multiplexed multi-point detection, and sensor networking ${ }^{3-6}$. However, fibre-based LAS sensors have limitations in performances. Conventional silica optical fibres have a low-loss transmission window from $\sim 0.5$ to $\sim 1.8 \mu \mathrm{m}$ and only allow access to the relatively weak overtone absorption lines of molecules, which significantly compromises the detection sensitivity. Taking gas detection as an example, fibre-pigtailed open-path and evanescent wave gas cells have demonstrated noise equivalent concentration (NEC) of $10-100$ p.p.m. ${ }^{3-6}$, with a linear dynamic range of two to three orders of magnitude ${ }^{7}$. Hollow-core photonic bandgap fibre (HC-PBF) gas cells enable stronger lightgas interaction over a longer distance and have demonstrated a NEC of $\sim 10$ p.p.m. methane with 5.1-m-long HC-PBF cells ${ }^{8}$, corresponding to a noise equivalent absorption coefficient (NEA) of $1.6 \times 10^{-6} \mathrm{~cm}^{-1}$. Further significant increase in detection sensitivity is challenging since it requires an impractically long length of $\mathrm{HC}-\mathrm{PBF}$, which also compromises the response time of the sensor significantly.

Instead of direct measurement of spectral attenuation, here we exploit photothermal (PT) effect in a HC-PBF and demonstrate ultra-sensitive gas detection by probing the gas-absorptioninduced phase change via fibre-optic interferometry. Optical absorption of molecules results in localized heating, which modifies the temperature, pressure and density, and modulates the refractive index (RI) of the material ${ }^{9}$. The RI modulation can be probed by a range of techniques such as PT lens $^{10}$, deflection ${ }^{11}$, diffraction ${ }^{12}$ and interferometry ${ }^{13,14}$. PT interferometry measures the change of optical phase accumulated over a propagation distance and has been demonstrated for detecting weak absorbance in gas- and liquidphase materials with very high sensitivity ${ }^{13-17}$. However, all the PT interferometry systems reported previously use free-space optics with an absorption distance up to several tens of centimetres, limited by beam divergence, optical loss and size of set-up. To access the stronger absorption lines, high-power gas lasers operating at the mid-infrared (MIR) were typically employed and they are complex, bulky and incompatible with fibre-optic systems.

In this article, we report an all-fibre PT interferometry system that uses a HC-PBF operating at the near infrared (NIR) wavelengths and demonstrate remarkably sensitive trace-gas detection with an unprecedented dynamic range. The HC-PBF confines the fluidic sample and propagating light modes simultaneously within the hollow-core, and the overlaps between the sample and the light fields approach $100 \%$ (refs 18-20). Light focused in a HC-PBF is much tighter and the interaction length is much longer, and the overall efficiency of light-sample interaction could be made significantly higher than a free-space PT system. This enables extremely high performance trace chemical detection systems operating at the NIR wavelengths, which are fully compatible with the telecom optical fibre technologies.

\section{Results}

Theory of PT spectroscopy in HC-PBF. The basics of PT spectroscopy in a HC-PBF may be explained with the aid of Fig. 1. When a wavelength/intensity modulated pump light beam
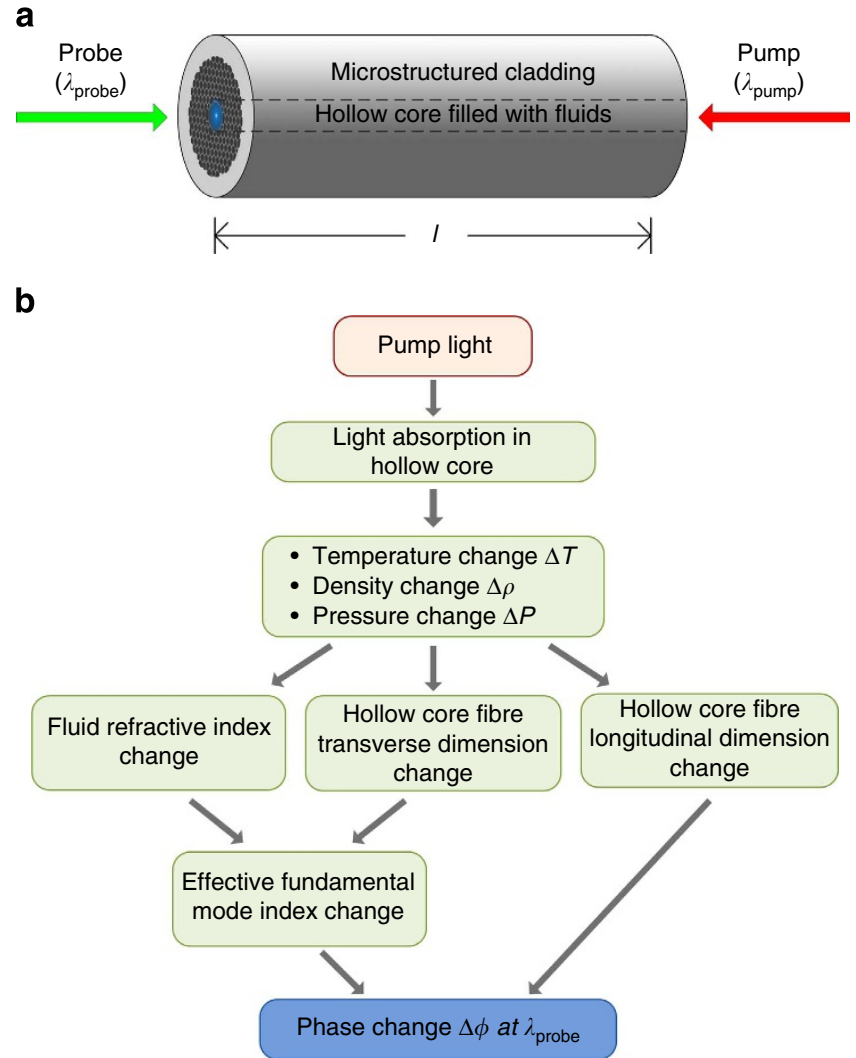

Figure 1 | PT-induced phase modulation in a HC-PBF. (a) Modulated pump beam $\left(\lambda_{\text {pump }}\right)$ and constant probe beam $\left(\lambda_{\text {probe }}\right)$ are counterpropagating in a fluid-filled HC-PBF. The pump and probe may also be arranged to co-propagate within the HC-PBF. (b) Processes involved in producing phase modulation in a HC-PBF.

is coupled into a fluid-filled HC-PBF, it interacts with the spectrally absorbing fluid; the fluid molecules are excited to higher energy states and then return to their initial state via molecular collision. This process is accompanied by periodic heat production, which modulates the local temperature, density and pressure, and hence the RI of the fluid. The temperature and pressure modulation also perturb the transverse and longitudinal dimensions of the $\mathrm{HC}-\mathrm{PBF}^{21,22}$. When a probe light beam is propagating along the same HC-PBF, the accumulated phase of the fundamental optical mode of the probe will be modulated.

Taking trace-gas detection as an example, for a pump beam propagating in a HC-PBF filled with a weakly absorbing gas, the intensity profile $I(r, z)$ of the fundamental mode may be approximated by a Gaussian distribution ${ }^{23}$

$$
I(r, z) \approx I_{0}(z) e^{-2 r^{2} / w^{2}}=\frac{2 P_{\text {pump }}(z)}{\pi w^{2}} e^{-2 r^{2} / w^{2}},
$$

where $I_{0}(z)$ is the pump light intensity at the centre of the fibre core, which decreases with propagation distance $z$ due to gas absorption and intrinsic fibre attenuation. $P_{\text {pump }}$ is the pump power and $2 w$ the mode field diameter (MFD), which has a value similar to the diameter of the hollow core. Assuming the heat yield is $Y_{H}$, the local heat production rate due to weak optical absorption may be expressed as ${ }^{9}$

$$
H(r, z)=Y_{H} A \bar{\chi}\left(\lambda_{\text {pump }}\right) I(r, z),
$$

where $\bar{\chi}(\lambda)$ is the peak normalized absorption line-shape function. $A=\alpha C$ is the peak absorption coefficient, $C$ the relative gas concentration and $\alpha$ the peak absorption coefficient for a relative concentration of $100 \%$. The heating process changes 
the temperature, density and pressure distribution of the fluid within the hollow core. The central portion of gas sample within the HC-PBF is heated up more, which reduces the density in the centre resulting in lower RI. This modulates the effective RI $\left(n_{\text {eff }}\right)$ of the fundamental mode as well as the length $(l)$ of the HC-PBF. To a first order approximation, the fractional change in effective RI of the fundamental mode $\left(\eta=\Delta \eta_{\text {eff }} / \eta_{\text {eff }}\right)$ and length $(\varepsilon=\Delta l / l)$ at location $z$ along the fibre may be expressed as

$$
\eta(z)+\varepsilon(z)=k A \bar{\chi}\left(\lambda_{\text {pump }}\right) I_{0}(z),
$$

where $k$ is a proportionality coefficient. The accumulated phase change $d \phi$ of the fundamental mode over interval $[z, z+d z]$ at the probe wavelength $\left(\lambda_{\text {probe }}\right)$ may then be expressed as

$$
d \phi=\frac{2 \pi n_{\text {eff }}}{\lambda_{\text {probe }}}[\eta(z)+\varepsilon(z)] d z .
$$

The phase change $\Delta \phi$ over a propagation distance $L$ may be obtained by integrating equation (4) and using equations (1) and (3), as

$$
\begin{aligned}
\Delta \phi & =\left(\frac{2 \pi n_{\text {eff }}}{\lambda_{\text {probe }}} \cdot \frac{2 k}{\pi w^{2}}\right) A \bar{\chi}\left(\lambda_{\text {pump }}\right) \cdot \int_{z=0}^{z=L} P_{\text {pump }}(z) d z \\
& =k^{*} A \bar{\chi}\left(\lambda_{\text {pump }}\right) \bar{P}_{\text {pump }} L,
\end{aligned}
$$

where $\bar{P}_{\text {pump }}$ represents the average pump power over length $L$ which, for weak absorption and short fibre length, equals approximately the pump power inputting into the HC-PBF. $k^{\star}$ is a coefficient proportional to $1 / w^{2}$. The phase modulation may be recovered accurately by use of highly sensitive fibre-optic interferometry.

Experimental set-up. The basic experimental set-up for the study of PT-induced phase modulation is shown in Fig. 2. The sensing HC-PBF is the NKT Photonics' HC-1550-02 fibre with hollow core diameter of $\sim 11 \mu \mathrm{m}$. A distributed feedback semiconductor pump laser is modulated at $50 \mathrm{kHz}$ and its wavelength is tuned to the $\mathrm{P}(9)$ line of acetylene $\left(\mathrm{C}_{2} \mathrm{H}_{2}\right)$ at $1,530.371 \mathrm{~nm}$. The acetylene molecular line strength at this wavelength is $1.211 \times 10^{-20} \mathrm{~cm}$ per molecule, corresponding to line strength of $0.3 \mathrm{~cm}^{-2} \mathrm{~atm}^{-1}$ at room temperature ${ }^{24}$. The peak absorption coefficient of the line for a relative concentration of $100 \%$ at room temperature (296 K) and $1 \mathrm{~atm}$ is then calculated to be $\alpha=1.165 \mathrm{~cm}^{-1}$. The HC-PBF forms the sensing arm of a fibre-optic Mach-Zehnder interferometer (MZI) while the reference arm is made of a standard single-mode fibre (SMF) coiled around a piezoelectric transducer (PZT). The PZT acts as a phase compensator to keep the MZI at quadrature via a servo-loop ${ }^{25}$ (see Supplementary Fig. 1 and Supplementary Discussion). The MZI is probed by an external-cavity diode-laser with its wavelength tuned to $1,556.59 \mathrm{~nm}$ where the absorption of acetylene is minimal. The MZI output (PD2) is demodulated by use of a lock-in amplifier and the output waveform can also be observed from an oscilloscope.

Test of lower detection limit. The capability of HC-PBF-based PT spectroscopy for detecting weak absorbance was tested with the set-up shown in Fig. 2. The sensing HC-PBF sample is $10-\mathrm{m}$ long and was fusion spliced to SMF-pigtails at both ends. Two microchannels were fabricated near the HC-PBF/SMF splicing joints for the purpose of pressurizing sample gas into the HC-PBF (see Methods). Figure 3a shows the second harmonic lock-in outputs for different pump power levels delivered to the HC-PBF when the pump wavelength was tuned across the $\mathrm{P}(9)$ line of acetylene in the $v_{1}+v_{3}$ band and the HC-PBF was filled with 10 p.p.m. acetylene balanced by nitrogen $\left(\mathrm{N}_{2}\right)$. For the pump power of $15.3 \mathrm{~mW}$, corresponding to a peak pump intensity of $I_{0}=40 \mathrm{~kW} \mathrm{~cm}^{-2}$, the peak-to-peak amplitude of the second harmonic signal is $\sim 1.37 \mathrm{mV}$. The second harmonic lock-in output when the pump is tuned away from the absorption peak to $1,530.53 \mathrm{~nm}$ is shown in Fig. 3b. The s.d. of the noise over 2-min duration is $0.26 \mu \mathrm{V}$, not much larger than the noise level $(0.24 \mu \mathrm{V})$ when the pump is off. However, a constant bias exists when the pump is on, due to background absorption. The lower detection limit for a signal to noise ratio of unity is estimated to be 2 p.p.b. in NEC and $2.3 \times 10^{-9} \mathrm{~cm}^{-1}$ in NEA, for a detection bandwidth of $0.094 \mathrm{~Hz}$.

The second harmonic signal and the s.d. of the noise as functions of pump power level are shown in Fig. 3c. The signal amplitude increases approximately linearly with pump power while the noise level showed very little change. The $1 \sigma$ NEC is better than 6 p.p.b. for pump power above $6 \mathrm{~mW}$. This shows that

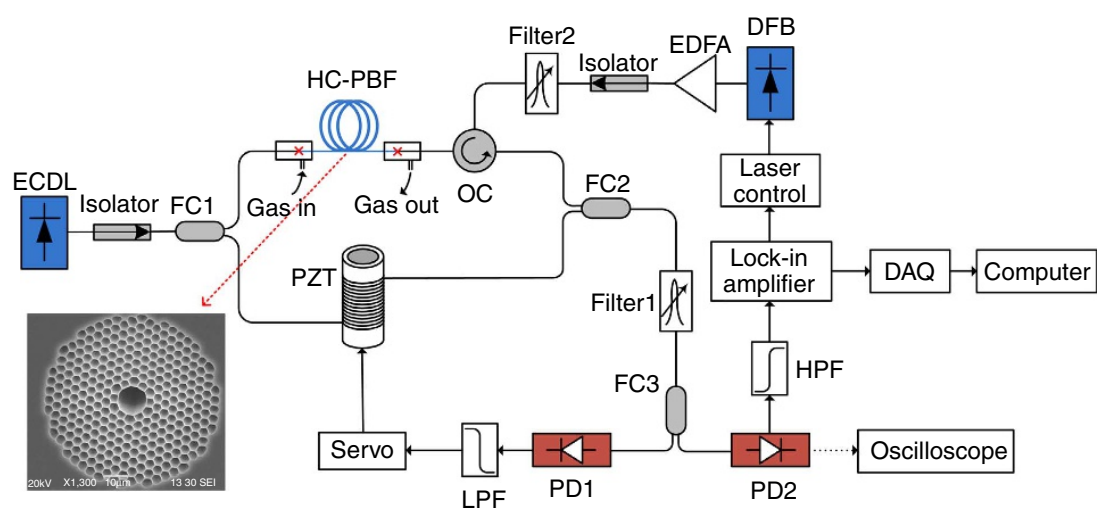

Figure 2 | Experimental set-up for gas detection with 10-m-long HC-PBF. The splitting ratios of FC1 and FC2 are, respectively, 80/20 and 50/50, which approximately balanced the powers in the two arms of the interferometer. The optical path lengths of the two arms are also balanced to minimize the laser phase to intensity noise conversation. Filter 1 is used to filter out the residual pump and Filter 2 to minimize the effect of EDFA's ASE noise. The splitting ratio of FC3 is 90/10. Output from PD1 passes a low-pass-filter (LPF) and is used for interferometer stabilization. Output from PD2 contains the PT-induced phase modulation signal. The driving current of the DFB is modulated at $50 \mathrm{kHz}$ by use of the internal signal generator of the lock-in. Inset: scanning electron microscopy (SEM) image of the HC-1550-02 fibre's cross-section. DAQ, data acquisition; DFB, distributed feedback laser (the pump); ECDL, external-cavity diode laser (the probe); EDFA, erbium-doped fibre amplifier; FC1-FC3, fibre couplers; OC, optical circulator; PD1-PD2, photo-detectors; PZT, piezoelectric transducer. 


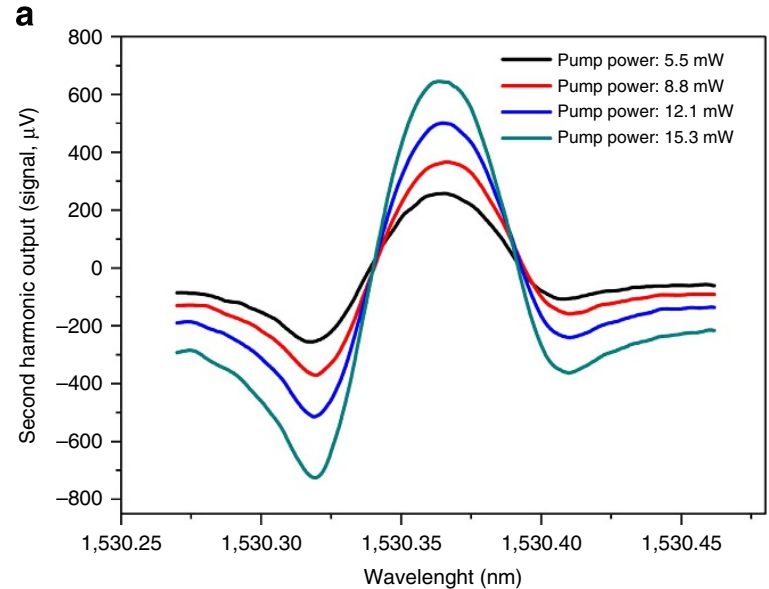

b

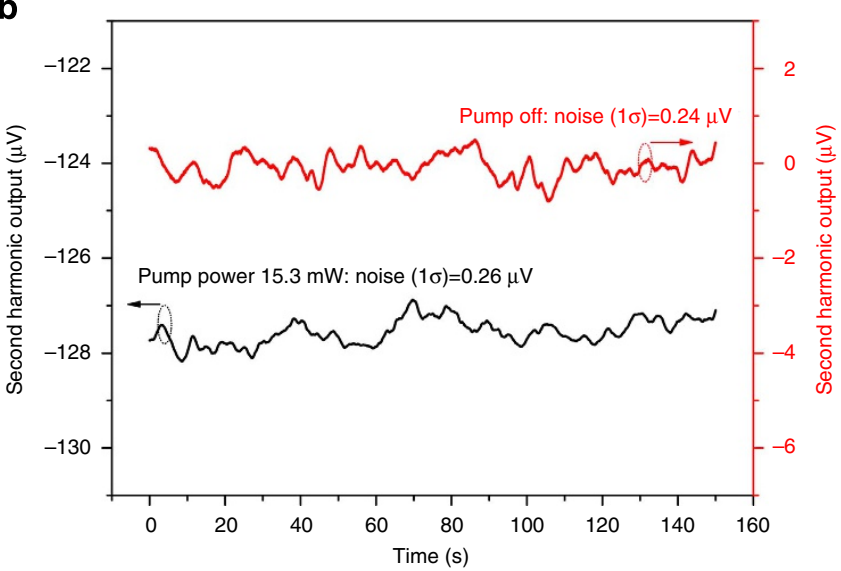

C

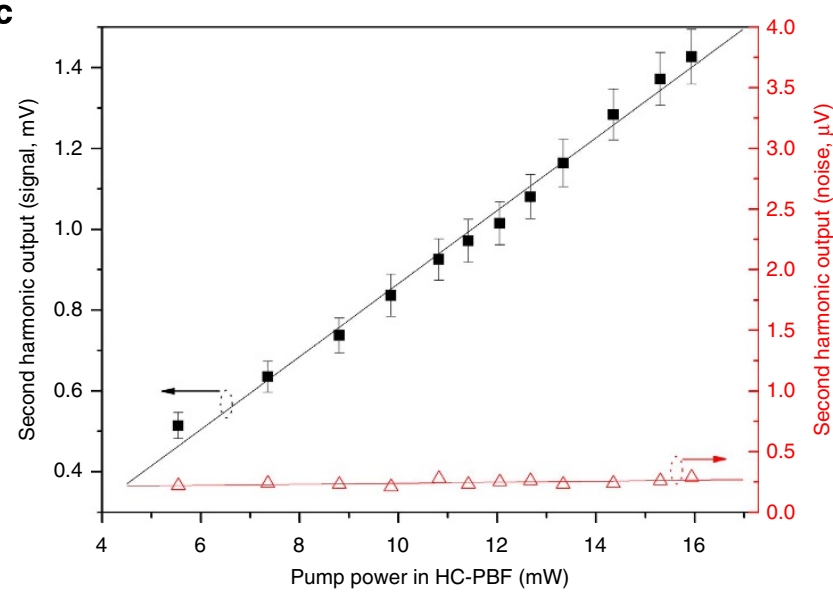

Figure 3 | Experimental results for 10-m-long HC-PBF. (a) Second harmonic lock-in output (signal) when pump laser is tuned across the $\mathrm{P}(9$ ) line of acetylene at $1,530.371 \mathrm{~nm}$. (b) Second harmonic lock-in output when the pump wavelength is fixed to $1,530.53 \mathrm{~nm}$. Black line: pump power is $\sim 15.3 \mathrm{~mW}$. Red line: pump power is zero (off). The measurements were conducted consecutively for the two pump power levels but the results are displayed in the same panel for easy comparison. (c) Second harmonic signal and the s.d. of the noise as functions of pump power level. Error bars show the s.d. from five measurements and the magnitudes of the error bars are scaled up by 10 -fold for clarity reason. The mean probe power level on PD2 is $\sim 50 \mu \mathrm{W}$. The gas concentration used is 10 p.p.m. acetylene balanced by nitrogen, and the experiments were conducted at room temperature. The amplitude of wavelength modulation was set to $\sim 2.2$ times of the absorption linewidth. The time constant of the lock-in amplifier is $1 \mathrm{~s}$ with a filter slope of $18 \mathrm{~dB} \mathrm{Oct}^{-1}$, corresponding to a detection bandwidth of $0.094 \mathrm{~Hz}$. p.p.b. level (acetylene) gas detection can be easily obtained with a relatively low power pump level, which is significantly better than the previously reported fibre-based LAS sensors.

It should be noted that the HC-PBF used in our experiment is not a true $\mathrm{SMF}^{26}$. It actually supports several higher-order modes and mode interference (MI) was identified as a major source of noise for the HC-PBF-based direct absorption sensors ${ }^{6}$. However, the MI was found to have minimal effect on the performance of the current HC-PBF-based PT sensor, evident from the very smooth trace of the second harmonic output as shown in Fig. 3a.

A closer examination of PT phase modulation and detection processes reveals that several factors would be responsible for the reduction of the MI. First, for the HC-PBF samples we used the cladding modes have already largely been removed. The first group of the higher-order mode (that is, $\mathrm{LP}_{11}$ ) still exists at the exit of the HC-PBF sample, but the power is relative small. For the 10 -m-long sample, the power in the $\mathrm{LP}_{11}$ mode at the output end is estimated to be $\sim 0.03 \%$ of the fundamental mode. Second, the probe wavelength is fixed and the probe interferometer is servo-locked to the quadrature point. The residual high order mode of the probe beam, which interferences with the fundamental modes in the HC-PBF and the SMF in the reference arm, would only cause slow intensity fluctuation at the interferometer output. The fluctuation is small in amplitude and would translate into, via servo-control, a small shift of the operating point and should have minimal effect on the detection of the higher frequency PT-induced phase modulation. Third, the pump beam is filtered out before reaching the photodetectors, and more importantly the higher-order mode power of the pump would have minimal effect on the phase modulation of the fundamental mode. This is because the change of effective RI of a propagating core mode due to perturbation of core material (gas) $\mathrm{RI}$ is proportional to $\int_{A}(n-\bar{n}) \bar{\Psi}^{2} d A$, where $n$ and $\bar{n}$ represent, respectively, the RI distribution for perturbed and unperturbed fibres, $\bar{\Psi}$ is mode field profile ${ }^{27}$. The integration is over the entire cross-section of the fibre. Since the induced RI change in gas is mainly due to density change, which tends to follow the intensity profile (heating profile) of the mode, the pump power in a higherorder mode tends to induce a RI perturbation that is 'orthogonal' to the profile of the fundamental mode. More specifically, the $\mathrm{LP}_{11}$ mode has a two-lobe intensity profile, which results in a reduction of gas RI near the lobe intensity maxima and an increase of gas RI at the centre of the core. Such a RI perturbation would make the overlap integral with the fundamental mode approaching zero and hence have minimal effect on the effective RI of the fundamental mode. For the same reason, the effect of pump power in the fundamental mode would also have minimal effect on the effective RI of the higher-order mode. This means that the effective RI (and hence phase of the probe) of a mode is only significantly modulated by the pump power in the same mode, and the cross-modulation due to pump power in a different mode would be minimal. These and the fact that only a very small fraction of pump and probe power is in the higherorder mode suggest that the effect of the higher-order mode would have minimal effect on the performance of the HC-PBFbased PT spectroscopic sensors. However, further detailed modelling and experimental investigations are needed to quantify the influence of the higher-order mode, which could be a challenging task due to the complex fluid/thermal dynamic processes in gases and the surrounding fibre microstructure.

Environmental disturbance (for example, temperature and strain) affects the phase difference between the two interfering beams of MZI and hence the operating point. At laboratory environment, the disturbance is small, and, at low frequencies, our PZT-servo-loop worked well and the quadrature operating 
a

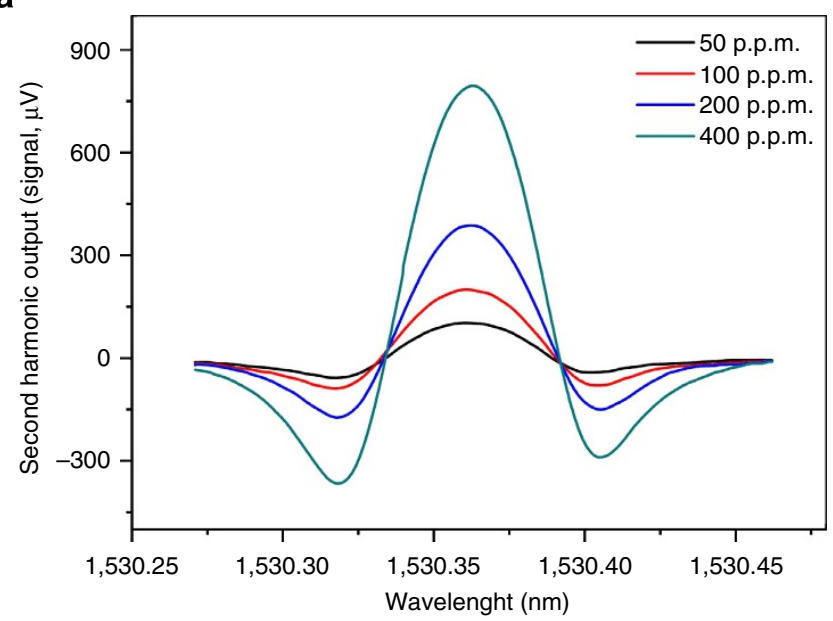

b

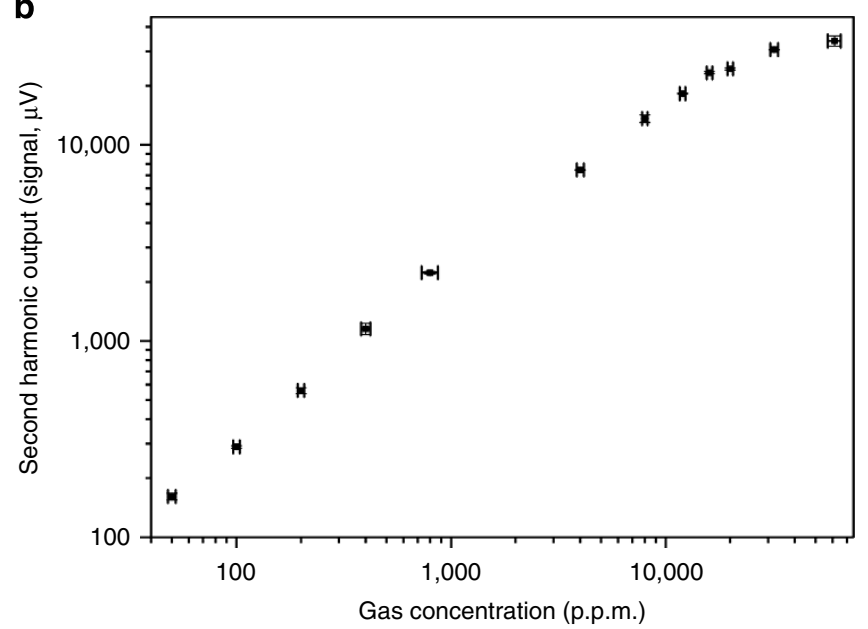

Figure 4 | Second harmonic signal as function of gas concentration.

(a) Second harmonic lock-in output signal when pump laser is tuned across the $P(9)$ line of acetylene at 1,530.371 nm for 50, 100, 200, 400 p.p.m. acetylene concentration. (b) Second harmonic signal (peak-to-peak value) as function of gas concentration. Error bars in the horizontal axis are based on the accuracy of the two mass flow controllers we used to prepare different gas concentrations. Error bars in the vertical axis show the s.d. from five measurements and the magnitudes of the error bars are scaled up by 10 -fold for clarity reason. The pump power in the hollow-core was estimated to be $\sim 25 \mathrm{~mW}$ and the mean probe power level on PD2 is $\sim 200 \mu \mathrm{W}$. The time constant of the lock-in amplifier is $1 \mathrm{~s}$ with a filter slope of $18 \mathrm{~dB} \mathrm{Oct}^{-1}$, corresponding to a detection bandwidth of $0.094 \mathrm{~Hz}$. The length of the sensing HC-PBF is $0.62 \mathrm{~m}$.

point is maintained well. The PT signal, which is at a much higher frequency $(50 \mathrm{kHz})$, was found not significantly affected by the drift of the operating point. For field applications, the environmental disturbance could be unpredictable and the ability of maintaining quadrature operation for the current MachZehnder scheme still needs to be tested. The fluctuation of the second harmonic output (see Fig. 3b) when the pump wavelength is tuned away from absorption peak was found being correlated with the output from PD1, which is used for MZI stabilization. This suggests that the active digital PZT servo is responsible for the noise level observed. The digital servo signal applied to the PZT introduces noise over a wide frequency range and the noise power within the system detection bandwidth is indistinguishable from the PT phase modulation signal, which sets the limit to concentration detection. The use of a passive phase demodulation

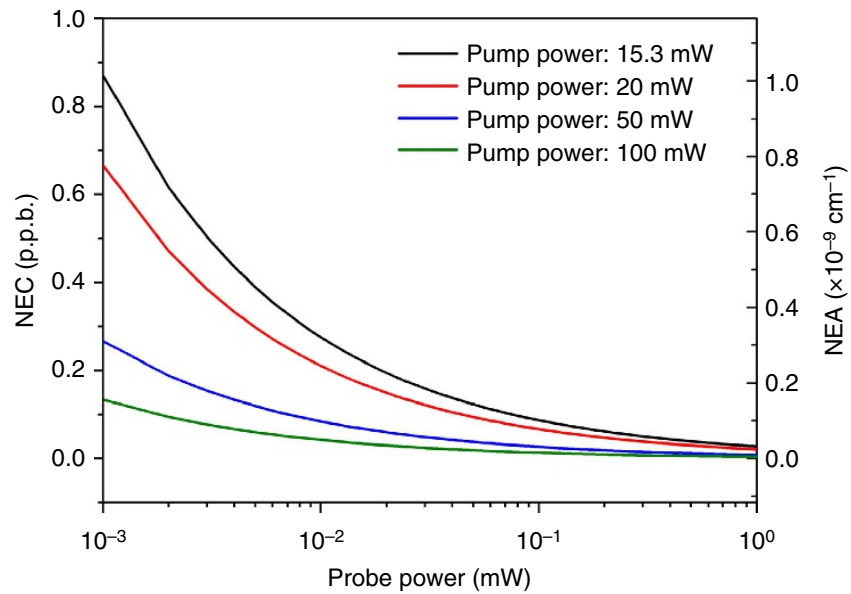

Figure 5 | Shot-noise limit in terms of NEC and NEA for different pump and probe power levels. The pump wavelength is tuned to the $P(9)$ absorption line of acetylene at $1,530.371 \mathrm{~nm}$ and the length of HC-PBF is $10 \mathrm{~m}$. Detection bandwidth is $0.094 \mathrm{~Hz}$, corresponding to $1 \mathrm{~s}$ time constant of the lock-in with a filter slope of $18 \mathrm{~dB}$ Oct $^{-1}$. Probe wavelength

$\lambda_{\text {probe }}=1,556.59 \mathrm{~nm}, v_{\text {probe }}=c / \lambda_{\text {probe. }}$. Detector quantum efficiency $\eta=0.8$, Planck constant $h=6.626 \times 10^{-34} \mathrm{~J} \mathrm{~s}$.

scheme in a Sagnac loop configuration would improve the performance of the system ${ }^{28}$.

Test of dynamic range. The dynamic range of the HC-PBF-based PT spectroscopic system was tested with a 0.62-m-long HC-PBF. The experimental set-up used is slightly different from Fig. 2 (see Supplementary Fig. 2). The HC-PBF was spliced to SMF-pigtails while multiple microchannels were drilled into the hollow core of the HC-PBF (see Methods) for easy filling of the hollow core with different gas concentrations without the need for pressurization. Figure 4 shows the second harmonic lock-in output for acetylene concentration from $\sim 50$ p.p.m. to $\sim 6 \%$ acetylene balanced with nitrogen. The lower acetylene concentrations (that is, 50 to 794 p.p.m.) were prepared by mixing pure nitrogen with $1 \%$ acetylene, while the higher concentrations were prepared by mixing nitrogen with $99.99 \%$ acetylene. An approximately linear relationship is obtained for acetylene concentration up to $\sim 1.6 \%$ and non-linearity starts to appear beyond this value. The lower detection limit in terms of $1 \sigma$ NEC is estimated to be 30 p.p.b. $\left(3.5 \times 10^{-8} \mathrm{~cm}^{-1}\right.$ in terms of NEA), giving a dynamic range of nearly six orders of magnitude $\left(5.3 \times 10^{5}\right)$. Such a high performance has not been achieved with any fibre-based gas detection system reported previously.

The dynamic range of the PT sensor would be limited by the non-linear transfer function of the interferometer and the non-linear dependence of the phase modulation beyond the weak absorption approximation. For the experimentally used pump modulation frequency of $50 \mathrm{kHz}$, the scale factor $k^{*}$, which is defined in equation (5), was determined to be $1.12 \mathrm{rad} \mathrm{cm} \mathrm{mW}^{-1} \mathrm{~m}^{-1}$ (see Methods). For the results shown in Fig. 4, which used $25 \mathrm{~mW}$ pump power and 0.62 -m-long HC-PBF sample, the phase shift at $1.6 \%$ acetylene concentration is calculated to be $\sim 0.233 \mathrm{rad}$, which is well within the $1 \%$ linear dynamic range of the interferometer transfer function (that is, $\Delta \phi \leq 0.1 \pi$ ). However, the pump power is absorbed significantly and reduced to $\sim 30 \%$ at the output of the HC-PBF sample, suggesting that the weak absorption assumption is no longer valid and the heat generation rate should follow an exponential function of the absorbance which is the factor responsible for the non-linear behaviour for larger acetylene concentrations. 
Table 1 | Detection limits of absorption-based optical fibre gas sensors.

\begin{tabular}{|c|c|c|c|c|c|c|}
\hline Gas type & $\begin{array}{c}\text { Wavelength } \\
(\mu \mathrm{m})\end{array}$ & Gas cell & Technique & Integration time (s) & NEC (p.p.m.) & $\begin{array}{c}\text { NEA } \\
\left(\mathrm{cm}^{-1}\right)\end{array}$ \\
\hline Methane 31 & 1.666 & $13.7 \mathrm{~cm} \mathrm{HC}-\mathrm{PBF}$ & WMS & Not stated & 158 & $1.8 \times 10^{-5 \star}$ \\
\hline Acetylene ${ }^{32}$ & 1.52 & $1 \mathrm{~m} \mathrm{HC}$-PBF reference cell, $14 \mathrm{~cm}$ measurement cell & CS & 4 & 100 & $1.3 \times 10^{-4 \star}$ \\
\hline Acetylene $^{33}$ & 1.53037 & $27 \mathrm{~m} \mathrm{HC}-\mathrm{PBF}$ & DAS & Not stated & 50 & $5.8 \times 10^{-5 \star}$ \\
\hline
\end{tabular}

CS, correlation spectroscopy; DAS, direct absorption spectroscopy; WMS, wavelength modulation spectroscopy

${ }^{\star}$ NEA data were calculated from NEC data and the absorption line strength from HITRAN database ${ }^{24}$.

Shot-noise-limited performance. The lower detection limit due to shot-noise may be estimated by ${ }^{15}$

$$
A_{\text {min }}=\frac{\Delta \phi_{\text {min }}}{k^{*} L \bar{P}_{\text {pump }}}=\frac{1}{k^{*} L \bar{P}_{\text {pump }}} \sqrt{\frac{2 h v_{\text {probe }} B}{\eta P_{\text {probe }}}},
$$

where $h$ is the Planck constant, $B$ the detection bandwidth and $\eta$ the efficiency of the photodetector. $v_{\text {probe }}$ and $P_{\text {probe }}$ are, respectively, the frequency and power of the probe beam. With equation (6) and the parameters used in our experiments, the shot-noise-limited detection sensitivity was calculated and shown in Fig. 5. For the $15.3 \mathrm{~mW}$ pump power, $50 \mu \mathrm{W}$ probe power, 10-m-long HC-PBF, and a detection bandwidth of $0.094 \mathrm{~Hz}$, the shot-noise-limited NEC and NEA are, respectively, 120 p.p.t. acetylene and $1.4 \times 10^{-10} \mathrm{~cm}^{-1}$. It should be mentioned that the above shot-noise calculation did not consider the effect of wavelength modulation. In our experiment, we used wavelength modulation and second harmonic detection, which would deteriorate the noise limit by a factor ${ }^{29}$ of $\sim 4$ and give an estimated detection limit of 480 p.p.t. NEC or $5.6 \times 10^{-10} \mathrm{~cm}^{-1}$ NEA. The calculated shot-noise-limited NEC is $\sim 4$ times better than the experimentally obtained detection limit.

\section{Discussion}

In summary, we have developed a novel all-fibre optical sensing technique based on PT phase modulation in a HC-PBF, which will enable high precision cost-effective fibre-optic sensors for real-time trace chemical detection. Preliminary experiments with acetylene gas demonstrated p.p.b. level detection limit and an extremely large linear dynamic range of nearly six orders of magnitude.

For comparison, we list in Table 1 the lower detection limits of some previously reported direct absorption fibre-optic gas sensors. Although the results were obtained with different gases, system configurations and parameters ${ }^{8,30-34}$, we may, however, compare the performance of these sensors in terms of NEA. With 5.1-m-long absorption path, the HC-PBF-based sensor demonstrated a NEA of $1.6 \times 10^{-6} \mathrm{~cm}^{-1}$, which is $\sim 700$ times worse than the performance of our current HC-PBF PT sensor.

With similar parameters as used in the PT sensor, we calculated the shot-noise limit of a direct absorption sensor for $50 \mu \mathrm{W}$ optical power reaching the photodetector and found to be 20 p.p.t. in terms of NEC or $2.3 \times 10^{-11} \mathrm{~cm}^{-1}$ in NEA. Again, the detection limit would be worsening by a factor of 4 if wavelength modulation/second harmonic detection is used ${ }^{29}$. These values are $\sim 6$ times smaller than the shot-noise limit of our PT sensor with $15.3 \mathrm{~mW}$ pump power, showing that the performances of current direct absorption optical fibre sensors are far away from their shot-noise limits. Current commercial $\mathrm{HC}-\mathrm{PBF}$ are not true $\mathrm{SMFs}^{26}$, and $\mathrm{MI}$ is a major factor that limits the performance of the HC-PBF-based direct absorption sensors ${ }^{6}$. However, MI has negligible effect on the performance of our HC-PBF-based PT spectroscopic sensors.
Similar to tunable diode LAS, the diode laser-based PT spectroscopic sensor could be made self-calibrating against intensity changes in the pump and probe. Assuming the interferometer is maintained at quadrature, the intensity modulation at the interferometer output would be linearly proportional to the absorption-induced phase change, that is,

$$
\Delta I=k_{e} P_{\text {probe }} \Delta \phi=k_{e} k^{*} P_{\text {probe }} \bar{P}_{\text {pump }} \alpha \bar{\chi}\left(\lambda_{\text {pump }}\right) C L,
$$

Where $k_{e}$ is a constant and $k_{e} P_{\text {probe }}$ is the proportionality factor related to interferometric detection and $k^{*} \bar{P}_{\text {pump }} \alpha \bar{\chi}\left(\lambda_{\text {pump }}\right) L$ is a factor related to the PT phase modulation. $k_{e} P_{\text {probe }}$ could in principle be self-calibrated by for example normalizing it against the DC component or peak-to-peak magnitude of the interferometer output. $k^{*} \bar{P}_{\text {pump }}$ could also be calibrated by detecting the residual intensity modulation of pump, similar to that for the direct absorption sensors ${ }^{35}$. The combination of the two would allow self-calibration of the PT HC-PBF sensor against both pump and probe power variations.

Compared with free-space PT interferometry systems, the use of HC-PBF offers significant benefits. First, the pump, probe and sample overlap perfectly within the hollow core, and the efficiency of sample excitation and probing is extremely high. Second, the MFDs of the fundamental mode of the state-of-theart commercial HC-PBFs are as small as $5-10 \mu \mathrm{m}$ (refs 18-20), over two orders of magnitude smaller than the diameter of a typical free-space beam $(1-2 \mathrm{~mm})^{13-17}$. This means that for the same power level, the pump intensity in a HC-PBF can be over four orders of magnitude higher, which significantly enhances the heat production. In contrast, to achieve the same pump intensity, the pump laser power required is much less, allowing the use of a lower cost pump source. Third, the fundamental mode in a HCPBF is well confined within the hollow core with low loss over a distance of metres to kilometres, and the absorption distance can then be 10 to $10^{4}$ times longer than a free-space system. The reduced beam size and increased absorption distance resulted from using HC-PBF would possibly enhance the overall efficiency of the PT phase modulation by 5 to 8 orders of magnitude, which would enable highly efficient PT fibre-optic devices. However, the thermal/fluid dynamic processes in a gas-filled HC-PBF could be very complex and significantly different from a free-space system; the efficiency of PT modulation would also depend on the modulation frequency. Further work is needed to understand the various processes involved and evaluate the full potential of the HC-PBF-based PT interferometric devices.

Current HC-PBFs are primarily operating in the visible and NIR where molecular absorptions of most gases and analytes are weaker than that in the MIR. However, the overall significantly enhanced efficiency as discussed above would compensate the signal reduction due to the weaker absorption. Operating in the NIR also allows the use of cheaper and more compact photonic components compatible with standard telecom optical fibres, making it possible to develop cost-effective all-fibre phase 
modulation devices, and remote, multiplexed and distributed sensing systems.

HC-PBF-based PT spectroscopy can readily be applied to detect other types of gases. The HC-1550-02 fibre we used in this investigation has a transmission window from 1,500 to $1,700 \mathrm{~nm}$, which covers a range of gases such as $\mathrm{CO}_{2}, \mathrm{CO}, \mathrm{CH}_{4}, \mathrm{~N}_{2} \mathrm{O}, \mathrm{H}_{2} \mathrm{~S}$, $\mathrm{NH}_{3}, \mathrm{HI}, \mathrm{C}_{4} \mathrm{H}_{6}$ and could then be used to detect these gases. Furthermore, there are a range of HC-PBFs with transmission bands from $400 \mathrm{~nm}$ to $2 \mu \mathrm{m}$ available from NKT Photonics, allowing a wider range of gas species to be detected.

The extension of HC-PBF-based PT spectroscopy to liquid analysis is straightforward. When combined with fibre-based microfluidic arrangement ${ }^{34,36}$, the technique would provide an ultra-sensitive means for environmental, chemical and biochemical detection with micro/nano litre sample consumption. The absorption-induced PT phase modulation in HC-PBF would allow novel all-fibre photonic devices, fluid sensing networks ${ }^{5}$ and distributed sensing system based on detecting phase modulation along the entire length of HC-PBF with, for example, coherent phase optical-time-domain-reflectometry ${ }^{37}$. The work could also be extended to other microstructured fibres such as suspendedcore and Kagome fibres and to other wavelength regions from ultra-violet to $\mathrm{MIR}^{34,38}$. The ability for transmitting highintensity laser beams within HC-PBFs could also be exploited for ultra-sensitive nonlinear fibre-optic PT spectroscopy ${ }^{39}$.

\section{Methods}

Preparation of HC-PBF samples. The fibre samples were prepared by splicing HC-1550-02 fibres to SMFs with the Ericsson FSU975 fusion splicer. The fusion duration was set to $0.2 \mathrm{~s}$, and the offset and overlap were 45 and $10 \mu \mathrm{m}$, respectively.

For the 0.62-m sample, the output end of the HC-1550-02 was fusion spliced to SMF with low fusion current to prevent the collapse of air-holes ${ }^{40}$. The input end was, however, fusion spliced to SMF with a larger fusion current of $\sim 16 \mathrm{~mA}$. This collapses the cladding air-holes to some extent and helps to minimize the excitation of the cladding modes and hence reduces modal interference (see Supplementary Fig. 3 and Supplementary Discussion). Fifteen microchannels were drilled along the sample by use of an $800-\mathrm{nm}$ femtosecond laser ${ }^{6}$, and the spacing between the microchannels ranges from 2 to $7 \mathrm{~cm}$. The average loss over 1,500 to $1,600 \mathrm{~nm}$ was estimated to be $\sim 0.02 \mathrm{~dB}$ per channel, and the total loss from the input to output SMF is $\sim 4 \mathrm{~dB}$. The drilling of microchannels was found not significantly increasing the MI (see Supplementary Fig. 4 and Supplementary Discussion).

The diameter of the microchannels is $\sim 5 \mu \mathrm{m}$, as shown in the SEM image in the Supplementary Fig. 4. At atmospheric pressure, the Knudsen number of the target gas is much smaller than 1 , as the mean free path of the gas molecule is much smaller than the diameter of the hollow core. The diffusion process is close to the continuum state and the microchannels with depth of $\sim 60 \mu \mathrm{m}$ do not affect the speed of gas diffusion significantly.

For the 10-m sample, the HC-PBF was fusion spliced to SMFs at both ends with a fusion current of $12.5 \mathrm{~mA}$. Compared with the $0.62-\mathrm{m}$ sample, the cladding modes are almost completely attenuated and the magnitudes of higher-order core modes are also reduced considerably $(\sim 0.03 \%$ of the fundamental mode power) because of the relatively longer length of HC-PBF used. Two microchannels were drilled from aside of the HC-PBF near the HC-PBF/SMF splicing joints and the total loss from the input to output SMF is $\sim 4 \mathrm{~dB}$.

Gas detection with the 10-m-long HC-PBF. The experimental setp-up used for the $10-\mathrm{m}$-long HC-PBF sample is shown in Fig. 2. The sections of the $10-\mathrm{m}$-long $\mathrm{HC}-\mathrm{PBF}$ with microchannels were placed within two $10-\mathrm{cm}$-long tubes with diameter of $1 \mathrm{~cm}$. One of the tubes was connected to a high-pressure gas cylinder containing 10 p.p.m. acetylene balanced by nitrogen while the other left open to atmosphere. The HC-PBF was first filled with the 10 p.p.m. acetylene with $\sim 2.5$ bar pressure for $\sim 2 \mathrm{~h}$, and then depressurized and sealed before taking measurements. The experiment was conducted at room temperature.

The pump power delivered to the HC-PBF was estimated by detecting pump power at the output of Filter 2, deducting the losses of the circulator and the SMF/HC-PBF splice.

To estimate the noise level, the second harmonic lock-in output when the pump was tuned to $1,530.53 \mathrm{~nm}$ (away from acetylene absorption peaks) was sampled at the rate of 40 samples per second and recorded, and the s.d. of the noise calculated.

Gas detection with the 0.62-m-long HC-PBF. The 0.62-m-long HC-PBF was placed inside a $23 \mathrm{~cm} \times 15 \mathrm{~cm} \times 7 \mathrm{~cm}$ gas chamber, and the sample gas with different concentrations was prepared by mixing 1 or $99.99 \%$ acetylene with pure nitrogen at different flow-rate ratios at atmospheric pressure through two mass flow controllers. For preparing the 50, 100, 200, 400, 794 p.p.m. acetylene samples, the flow rates used are, respectively, 1, 2, 4, 5 and $5 \mathrm{sccm}$ (standard cubic centimetre per minute) of $1 \%$ acetylene with $199,198,196,120$ and $58 \mathrm{sccm}$ of pure nitrogen. For preparing the $0.4,0.8,1.2,1.6,2,3.205$ and $6.098 \%$ gas samples, the flow rates used are, respectively, 1, 2, 3, 4, 5, 5 and $5 \mathrm{sccm}$ of $99.99 \%$ acetylene with 249,248 $247,246,245,151$ and $77 \mathrm{sccm}$ of pure nitrogen. The sample gas diffuses into the hollow core through the microchannels made on the HC-PBF.

The experimental set-up used for the $0.62-\mathrm{m}$-long HC-PBF sample is similar to the one shown in Fig. 2, but the pump power is injected into the HC-PBF via one port of FC2 (see Supplementary Fig. 2). The methods used for calculating the pump power level to the $\mathrm{HC}-\mathrm{PBF}$ and the s.d. of the noise are similar to the $10-\mathrm{m}$ sample.

Determination of scale factor $\boldsymbol{k}^{\star}$. The 0.62 -m-long HC-PBF sample was filled with $1 \%$ acetylene, and the pump power input to the HC-PBF was estimated to be $35 \mathrm{~mW}$. The amplitude of wavelength modulation was set to $\sim 3.2$ times of the absorption linewidth and the centre wavelength of the pump was tuned to $1,530.371 \mathrm{~nm}$. The interference fringe contrast was estimated by observing the PD2 output with an oscilloscope as illustrated in Supplementary Fig. 3, and the maximum and minimum voltages without phase stabilization were first measured to be 0.2 and $2.9 \mathrm{~V}$. The peak-to-peak modulation signal from PD2 when the interferometer is stabilized at quadrature was determined to be $0.195 \mathrm{~V}$. By comparing this value with the fringe contrast, the amplitude of the induced phase modulation was determined to be $0.145 \mathrm{rad}$. Since the absorption of $1 \%$ acetylene is not weak, we calculated the average pump power by integrating the absorptioninduced power variation over the entire length of the HC-PBF and obtained $k^{*} \alpha=1.3 \times 10^{-6} \mathrm{rad}$ p.p.m. ${ }^{-1} \mathrm{~mW}^{-1} \mathrm{~m}^{-1}$ or $k^{*}=1.12 \mathrm{rad} \mathrm{cm} \mathrm{mW}^{-1} \mathrm{~m}^{-1}$

\section{References}

1. Werle, P. et al. Near- and mid-infrared laser-optical sensors for gas analysis. Opt. Laser Eng. 37, 101-114 (2002).

2. Willer, U., Saraji, M., Khorsandi, A., Geiser, P. \& Schade, W. Near- and mid-infrared laser monitoring of industrial processes, environment and security applications. Opt. Laser Eng. 44, 699-710 (2006).

3. Shemshad, J., Aminossadati, S. M. \& Kizil, M. S. A review of developments in near infrared methane detection based on tunable diode laser. Sensor. Actuat. B Chem. 171, 77-92 (2012).

4. Hodgkinson, J. \& Tatam, R. P. Optical gas sensing: a review. Meas. Sci. Technol. 24, 012004 (2013).

5. Culshaw, B., Stewart, G., Dong, F., Tandy, C. \& Moodie, D. Fibre optic techniques for remote spectroscopic methane detection - from concept to system realisation. Sensor. Actuat. B Chem. 51, 25-37 (1998).

6. Jin, W., Ho, H. L., Cao, Y. C., Ju, J. \& Qi, L. F. Gas detection with micro- and nano-engineered optical fibers. Opt. Fiber Technol. 19, 741-759 (2013).

7. Frish, M. B. et al. Precision and accuracy of miniature tunable diode laser absorption spectrometers. Proc. SPIE 8032, 803209 (2011).

8. Cubillas, A. M. et al. Methane detection at $1670-\mathrm{nm}$ band using a hollow-core photonic bandgap fiber and a multiline algorithm. Opt. Express 15, 17570-17576 (2007).

9. Bialkowski, S. E. Photothermal Spectroscopy Methods for Chemical Analysis (John Wiley \& Sons, 1996).

10. Liu, M. \& Franko, M. Progress in thermal lens spectrometry and its applications in microscale analytical devices. Crit. Rev. Anal. Chem. 44, 328-353 (2014).

11. Fournier, D., Boccara, A. C., Amer, N. M. \& Gerlach, R. Sensitive in situ trace-gas detection by photothermal deflection spectroscopy. Appl. Phys. Lett. 37, 519-521 (1980).

12. Bialkowski, S. E. \& Chartier, A. Diffraction effects in single- and two-laser photothermal lens spectroscopy. Appl. Opt. 36, 6711-6721 (1997).

13. Stone, J. Measurements of the absorption of light in low-loss liquids. J. Opt. Soc Am. 62, 327-333 (1972).

14. Davis, C. C. \& Petuchowski, S. J. Phase fluctuation optical heterodyne spectroscopy of gases. Appl. Opt. 20, 2539-2554 (1981).

15. Mazzoni, D. L. \& Davis, C. C. Trace detection of hydrazines by optical homodyne interferometry. Appl. Opt. 30, 756-764 (1991).

16. Weston, N. D., Sakthivel, P. \& Mukherjee, P. Ultrasensitive spectral trace detection of individual molecular components in an atmospheric binary mixture. Appl. Opt. 32, 828-835 (1993).

17. Owens, M. A., Davis, C. C. \& Dickerson, R. R. A photothermal interferometer for gas-phase ammonia detection. Anal. Chem. 71, 1391-1399 (1999).

18. Russell, P. Photonic crystal fibers. Science 299, 358-362 (2003).

19. Smith, C. M. et al. Low-loss hollow-core silica/air photonic bandgap fibre. Nature 424, 657-659 (2003).

20. Benabid, F., Couny, F., Knight, J. C., Birks, T. A. \& Russell, P. St. J. Compact, stable and efficient all-fibre gas cells using hollow-core photonic crystal fibres. Nature 434, 488-491 (2005).

21. Dangui, V., Kim, H. K., Digonnet, M. J. F. \& Kino, G. S. Phase sensitivity to temperature of the fundamental mode in air-guiding photonic-bandgap fibers. Opt. Express 13, 6669-6684 (2005). 
22. Cao, Y., Jin, W., Yang, F. \& Ho, H. L. Phase sensitivity of fundamental mode of hollow-core photonic bandgap fiber to internal gas pressure. Opt. Express 22, 13190-13201 (2014).

23. Digonnet, M. J. F., Kim, H. K., Kino, G. S. \& Fan, S. Understanding air-core photonic-bandgap fibers: analogy to conventional fibers. J. Lightwave Technol. 23, 4169-4177 (2005).

24. Rothman, L. S. et al. The HITRAN 2004 molecular spectroscopic database. J. Quant. Spectrosc. Radiat. Transfer 96, 139-204 (2005).

25. Rosenthal, A., Kellnberger, S., Sergiadis, G. \& Ntziachristos, V. Wideband fiber-interferometer stabilization with variable phase. IEEE Photon. Technol. Lett. 24, 1499-1501 (2012).

26. Fini, J. M. et al. Low-loss hollow-core fibers with improved single-modedness. Opt. Express 21, 6233-6242 (2013).

27. Synder, A. W. \& Love, J. D. Optical Waveguide Theory (Chapman \& Hall, 1983).

28. Kråkenes, K. \& Blфtekjaer, K. Sagnac interferometer for underwater sound detection: noise properties. Opt. Lett. 14, 1152-1154 (1989).

29. Ye, J., Ma, L. S. \& Hall, J. L. Ultrasensitive detections in atomic and molecular physics: demonstration in molecular overtone spectroscopy. J. Opt. Soc. Am. B 15, 6-15 (1998)

30. Morante, M. A., Stewart, G., Culshaw, B. \& Lopez-Higuera, J. M. New microoptic cell for optical fibre gas sensors with interferometric noise reduction. Electron. Lett. 33, 1407-1409 (1997).

31. Carvalho, J. P. Remote system for detection of low-levels of methane based on photonic crystal fibres and wavelength modulation spectroscopy. J. Sens. 2009, 398403 (2009).

32. Austin, E., van Brakel, A., Petrovich, M. N. \& Richardson, D. J. Fibre optical sensor for $\mathrm{C}_{2} \mathrm{H}_{2}$ gas using gas-filled photonic bandgap fibre reference cell. Sensor. Actuat. B Chem. 139, 30-34 (2009).

33. Wynne, R. M., Barabadi, B., Creedon, K. J. \& Ortega, A. Sub-minute response time of a hollow-core photonic bandgap fiber gas sensor. J. Lightwave Technol. 27, 1590-1596 (2009).

34. Cubillas, A. M. et al. Photonic crystal fibres for chemical sensing and photochemistry. Chem. Soc. Rev. 42, 8629-8648 (2013).

35. Jin, W., Xu, Y. Z., Demokan, M. S. \& Stewart, G. Investigation of interferometric noise in fiber-optic gas sensors with use of wavelength modulation spectroscopy. Appl. Opt. 36, 7239-7246 (1997).

36. Unterkofler, S. et al. Microfluidic integration of photonic crystal fibers for online photochemical reaction analysis. Opt. Lett. 37, 1952-1954 (2012).

37. Lu, Y., Zhu, T., Chen, L. \& Bao, X. Distributed vibration sensor based on coherent detection of phase-OTDR. J. Lightwave Technol. 28, 3243-3249 (2010).
38. Wheeler, N. V. et al. Low-loss and low-bend-sensitivity mid-infrared guidance in a hollow-core-photonic-bandgap fiber. Opt. Lett. 39, 295-298 (2014).

39. Zharov, V. P. Ultrasharp nonlinear photothermal and photoacoustic resonances and holes beyond the spectral limit. Nat. Photon 5, 110-116 (2011).

40. Xiao, L., Demokan, M. S., Jin, W., Wang, Y. \& Zhao, C.-L. Fusion splicing photonic crystal fibers and conventional single-mode fibers: microhole collapse effect. J. Lightwave Technol. 25, 3563-3574 (2007).

\section{Acknowledgements}

This work was supported by the National Natural Science Foundation of China through grant no. 61290313, the Hong Kong SAR government through a GRF grant PolyU 152064/14E, and The Hong Kong Polytechnic University through Grant No: G-YK62 and G-YN26. We thank Wa Jin, Chao Wang, Jun Ma, Yuechuan Lin, Yanzhen Tan, Yongqin $\mathrm{Yu}$ and Yang Liu for useful discussions.

\section{Author contributions}

W.J. proposed and coordinated the work. Y.C. jointly developed the idea, built the experimental set-up and carried out preliminary experiments. F.Y. and H.L.H. modified the set-up. F.Y. prepared the samples, conducted gas detection measurements and achieved the results reported. W.J. and F.Y. analysed the mode interference noise. We performed data analysis and drafted the manuscript together.

\section{Additional information}

Supplementary Information accompanies this paper at http://www.nature.com/ naturecommunications

Competing financial interests: The authors declare no competing financial interests.

Reprints and permission information is available online at http://npg.nature.com/ reprintsandpermissions/

How to cite this article: Jin, W. et al. Ultra-sensitive all-fibre photothermal spectroscopy with large dynamic range. Nat. Commun. 6:6767 doi: 10.1038/ncomms7767 (2015).

(c) (i) This work is licensed under a Creative Commons Attribution 4.0 International License. The images or other third party material in this article are included in the article's Creative Commons license, unless indicated otherwise in the credit line; if the material is not included under the Creative Commons license, users will need to obtain permission from the license holder to reproduce the material. To view a copy of this license, visit http://creativecommons.org/licenses/by/4.0/ 